\title{
Contrapulsação em operação cardíaca: análise retrospectiva da incidência de infecção
}

\author{
Luís Henrique Barbosa BORGES ${ }^{*}$, Luis Fernando A. CAMARGO*, Tânia Mara V. STRABELLI*, David
} E. UIP*, José Otávio C. AULER JÚNIOR*

BORGES, L. H. B.; CAMARGo, L. F. A.; STRABELLI, T. M. V.; UIP, D. E.; AULER JÚNIOR, J. O. C. Contrapulsação em operação cardiaca: análise retrospectiva da incidência de infecçāo. Rev. Bras. Cir. Cardiovasc., 9 (1): 88-94, 1994.

RESUMO: Recentes avanços tecnológicos ampliaram o uso do balão intra-aórtico como medida de suporte na insuficiência cardiaca aguda. Apesar disto, têm sido descritas algumas complicaçōes relacionadas à sua inserção, duraçāo do uso e localizaçāo. O objetivo deste estudo foi investigar retrospectivamente a ocorrência de infecçōes em pacientes críticos que necessitaram do uso do balāo intra-aórtico (BIA) após operação cardiaca. Entre janeiro de 1990 e julho de 1992, foram revisados os prontuários de 97 pacientes que necessitaram de BIA no pós-operatório de operação cardíaca, sendo que apenas 55 apresentavam informaçōes completas que permitiram sua inclusão na revisão. Foram obtidas informaçōes a respeito de ocorrência de infecçōes, resultados de culturas, tipo e tempo de duração das operaçōes, tempo de circulação extracorpórea, duração da cateterização intravascular e evoluçāo clinica. Foram considerados os seguintes locais de infecção: pulmão, urina, corrente sangũinea, ferida operatória e local de inserçāo do BIA. A média de permanência do BIA foi de 3,9 2 2,01 dias e os tempos médios de operação e de circulação extracorpórea foram $8 \mathrm{he} 2,5 \mathrm{~h}$, respectivamente. Observamos uma alta incidência de infecções nestes pacientes, principalmente pneumonia $(63,6 \%)$. A taxa de infecção no local de inserção do BIA foi de $7 \%$ e maior que a taxa geral de infecção da ferida operatória em nossa Instituição (3\%). Apesar desta alta incidência de infecçōes não relacionar-se diretamente com a taxa de mortalidade, sugerimos rigorosa vigilância com relaçāo à ocorrência de infecçōes e possivveis medidas profiláticas em relação a infecções pulmonares

DESCRITORES: balão intra-aórtico, complicaçōes, infecçōes; contrapulsação em cirurgia cardiaca.

\section{INTRODUÇÃO}

O balão intra-aórtico vem sendo amplamente utilizado desde 1967 nas síndromes de baixo débito, principalmente como terapêutica temporária, no infarto agudo do miocárdio, no pré-operatório de operação cardíaca e angioplastia coronária. Complicações, principalmente vasculares, hemorrágicas e infecciosas tornaram-se mais freqüentes com a ampliação do seu uso, com aumento do tempo de permanência e o maior tempo de sobrevida dos pacientes.
As infecções diretamente relacionadas à presença do balão intra-aórtico são as infecções no local de inserção e as bacteremias primárias, com vários autores ${ }^{2-8}$ relatando indices de $2,6 \%$ a $27 \%$. Entretanto, em função da gravidade clínica, estes pacientes são mais suscetíveis a outros eventos infecciosos, não necessariamente relacionados à presença do balão, como, por exemplo, pneumonias.

Pela observação clínica diária em nosso Serviço, constatamos que os pacientes cirúrgicos que

Trabalho realizado no Instituto do Coração do Hospital das Clínicas da Faculdade de Medicina da Universidade de Sẫo Paulo. São Paulo, SP. Brasil. Recebido para publicaçāo em abril de 1994.

- Do Instituto do Coraçāo do Hospital das Clínicas da Faculdade de Medicina da Universidade de São Paulo.

Endereço para correspondência: Luis F. A. Camargo, Av. Dr. Enéas de Carvalho Aguiar, 44, SEDEQ. 05403, São Paulo, SP, Brasil. 
BORGES, L. H. B.; CAMARGO, L. F. A.; STRABELLI, T. M. V.; UIP, D. E.; AULER JÚNIOR, J. O. C. - Contrapulsação em operaçāo cardiaca: análise retrospectiva da incidência de infecção. Rev. Bras. Cir. Cardiovasc., 9 (2): 88-94, 1994

necessitam de suporte circulatório por balão intraaórtico são freqüentemente mais graves, sofrendo abordagem mais invasiva para cuidados e controle dos seus parâmetros. Por este motivo, conduzimos estudo retrospectivo e descritivo sobre o comportamento das infecções hospitalares nesta população específica.

\section{CASUISTICA E MÉTODOS}

Foram revisados os prontuários de pacientes submetidos a operações cardiovasculares que necessitaram de BIA para suporte circulatório, de janeiro de 1990 a julho de 1992, na Unidade de pósoperatório do Instituto do Coração do Hospital das Clínicas da Faculdade de Medicina da Universidade de São Paulo. De 97 casos registrados neste período, 27 foram a óbito nas primeiras 24 horas após a instalação do BIA e foram excluídos da avaliação. Dos 70 pacientes restantes, apenas 55 foram incluídos no estudo, por conterem prontuários com informações completas. Esta população foi estudada quanto a presença de infecções, culturas de microrganismos, tipo e tempo de duração das operações, tempo de circulação extracorpórea, tipo e tempo de uso de cateteres intravasculares e evolução clínica.

Os dados gerais da população estudada estāo na Tabela 1. Predominou o sexo masculino, com média de idade de 58,7 anos. Quatorze $(25,5 \%)$ pacientes evoluíram para óbito.

Os dados sobre as operações implicadas estão nas Tabelas 2 e 3 . Houve predomínio de revascularização do miocárdio $(52 \%)$. Sessenta porcento das operações (33) foram eletivas. Ocorreram 10 reoperações (nova intervenção por complicação direta da primeira) e 40 pacientes apresentaram alguma complicação no intra-operatório (massagem cardíaca interna, massagem cardíaca externa, sangramento aumentado e/ou dificuldades para sair da circulação extracorpórea).

TABELA 1

CARACTERISTICAS GERAIS DOS PACIENTES QUE NECESSITARAM DE SUPORTE CIRCULATORIO POR BALÃO INTRA-AÓRTICO

$\begin{array}{ll}\begin{array}{l}\text { Média de idade } \\ \text { Sexo } \\ \text { Masculino }\end{array} & 58,7 \pm 2,01 \text { anos } \\ \quad \begin{array}{l}\text { Feminino } \\ \text { Tempo Médio de }\end{array} & 44(80 \%) \\ \text { Internação no Pré-operatório } & 11(20 \%) \\ \begin{array}{l}\text { Tempo Médio de } \\ \text { Internação no Pós-operatório }\end{array} & 10,2 \pm 10,2 \\ \begin{array}{l}\text { Número de Óbitos ainda internados } \\ \text { Número de Altas }\end{array} & 31,6 \pm 22,5 \text { dias } \\ & 14(25,5 \%) \\ & 41(74,5 \%)\end{array}$

TABELA 2

TIPOS DE OPERACOÕES REALIZADAS EM PACIENTES QUE NECESSITARAM DE BALÃO INTRA-AÓRTICO

\begin{tabular}{lc}
\hline OPERAÇĀO REALIZADA & $N(\%)$ \\
\hline Revascularização do Miocárdio & $29(52,7)$ \\
Operação Valvar & $6(10,9)$ \\
Transplante Cardiaco & $1(1,8)$ \\
Correçāo de Aneurisma de Aorta & $1(1,8)$ \\
Complicações Pós-Infarto & \\
Agudo do Miocárdio & $5(9,1)$ \\
Revascularização do & \\
Miocárdio + Operação Valvar & $6(10,9)$ \\
Revascularização do & \\
Miocárdio + Complicações Pós-IAM & $6(10,9)$ \\
Angioplastia & $1(1,8)$ \\
\hline
\end{tabular}

TABELA 3

DADOS GERAIS SOBRE AS OPERAÇÕES EM PACIENTES COM BALÄO INTRA-AORTICO

\begin{tabular}{lc}
\hline VARIÁVEL & VALOR \\
\hline Tempo Médio de & \\
Circulaçāo Extracorpórea & $143,3 \pm 62,2$ minutos \\
Tempo Médio de Sala Operatória & $468,5 \pm 122,9$ minutos \\
Complicações Intra-operatórias & $40(72,7 \%)$ \\
Reoperaçōes & $10(18,2 \%)$ \\
Operaçōes de Urgência & $22(40 \%)$ \\
\hline
\end{tabular}

O BIA foi colocado em artéria femoral por via percutânea em todos os casos. Na maioria dos pacientes, a colocação ocorreu durante o ato operatório (Tabela 4 ) e a média de permanência foi de $3,90 \pm 2,01$ dias. Os dados foram armazenados em banco de dados software D-BASE III. Com relação à análise estatística, utilizou-se o Teste $T$ de Student não-pareado para comparação entre variáveis quantitativas e o teste exato de Fisher para as variáveis qualitativas, quando da comparação entre pacientes infectados e não-infectados.

O teste de Kruskal-Wallis (variáveis quantitativas) e o teste exato de Fisher (variáveis qualitati-

TABELA 4

MOMENTO DA COLOCACÃO DO BALĀO INTRAAÓRTICO (BIA) EM RELAÇÃO Aं OPERAÇÃO

\begin{tabular}{lc}
\hline COLOCAÇÃO DO BIA & $N(\%)$ \\
\hline Durante o Ato Operatório & $36(65,5)$ \\
Pós-Operatório & $13(23,6)$ \\
Reoperação & $2(3,6)$ \\
Pré-Operatório & $4(7,3)$ \\
\hline
\end{tabular}


BORGES, L. H. B.; CAMARGO, L. F. A.; STRABELLI, T. M. V.; UIP, D. E.; AULER JÚNIOR, J. O. C. - Contrapulsaçāo em operaçāo cardiaca: análise retrospectiva da incidência de infecção. Rev. Bras. Cir. Cardiovasc., 9 (2): 88-94, 1994

vas) foram utilizados quando da comparação entre os grupos com bacteremia primária, sem bacteremia primária mas com outra infecção e o grupo sem infecção. O nível de significância de $5 \%$ foi estabelecido para a análise dos resultados.

O diagnóstico das infecções foi feito baseandose nos seguintes critérios:

1) Infecção pulmonar: presença de febre, leucocitose, aumento da quantidade de secreção traqueal ou mudança do aspecto da mes$\mathrm{ma}$, independente do aparecimento de imagem radiológica.

2) Infecção do trato urinário: presença de mais de 100.000 colônias de bactérias $/ \mathrm{ml}$ na urocultura, independente da sintomatologia; presença de menos de 100.000 unidades formadoras de colônias $/ \mathrm{ml}$ de bactérias, porém com sintomatologia sugestiva (disúria, polaciúria, urgência miccional).

3) Infecção da ferida operatória: presença de secreção purulenta em ferida operatória;

4) Infecção no local da inserção do BIA: presença de secreção purulenta local, com ou sem cultura positiva.

5) Infeç̧ão primária da corrente sangüinea: febre, leucocitose e instabilidade hemodinâmica sem foco infeccioso aparente e/ou parâmetros hemodinâmicos medidos ao cateter de SwanGanz sugestivos de sepsis (baixa resistência vascular sistêmica e indice cardíaco aumentado) sem uso de drogas vasoativas. A infecção primária da corrente sangüinea foi considerada provável quando não houve confirmação etiológica (hemocultura positiva) e definitiva quando houve a comprovação.

6) Infecção secundária da corrente sangüínea: presença de hemocultura positiva para agente considerado patogênico, na vigência de um foco infeccioso definido.

\section{RESULTADOS}

Quarenta e três $(78,2 \%)$ pacientes apresentaram algum tipo de infecção, num total de 49 eventos infecciosos (Tabela 5). Seis pacientes apresentaram mais de uma infecção, sendo a primeira diagnosticada em média 3,3 dias após a colocação do BIA. Entre os eventos infecciosos, houve predomínio $(80 \%)$ das infecções pulmonares, que ocorreram em $63,6 \%$ dos pacientes.

Com relação à infecção da corrente sangüínea, foram feitos 10 diagnósticos baseados nos critérios descritos previamente (Tabela 6). Não houve con-
TABELA 5

INFECCOÕES EM PACIENTES COM BALĀO INTRA-AÓRTICO

\begin{tabular}{lc}
\hline LOCAL DA INFECÇÃO & No DE PACIENTES (\%) \\
\hline Pulmão & $35(63,6 \%)$ \\
Urina & $2(3,6 \%)$ \\
Ferida de Inserção do BIA & $4(7,3 \%)$ \\
Ferida Operatória & $2(3,6 \%)$ \\
Bacteremia Primária & $6(10,9 \%)$ \\
\hline
\end{tabular}

firmação etiológica das seis bacteremias primárias diagnosticadas pelos critérios estabelecidos. As bacteremias secundárias $(n=4)$, tiveram como agentes isolados Enterobacter $s p$ em 1 caso, $S$. aureus em 1 caso e $S$. epidermidis em 2 casos. A média de permanência do BIA entre os 6 pacientes com bacteremia primária foi de 3,3 dias e o diagnóstico de infecção foi feito em média dois dias após a operação. Houve 2 óbitos neste grupo de pacientes.

TABELA 6

DADOS REFERENTES ÀS INFECÇŌES DE CORRENTE SANGÜINEA

\begin{tabular}{ll}
\hline BACTEREMIAS & N \\
\hline Primárias & 6 \\
Secundárias & 4 \\
Pulmōes & 2 \\
Ferida de Inserção do BIA & 1 \\
Ferida Operatória & 1 \\
\hline
\end{tabular}

Com relação à análise estatística, foram comparados os pacientes que apresentaram infecção com aqueles que evoluiram sem complicaçōes infecciosas, com o objetivo de se estabelecerem fatores de risco. Observou-se que as seguintes variáveis estiveram associadas à ocorrência de processos infecciosos em pacientes com BIA: duração da operação, tempo de uso do balão intra-aórtico e tempo de uso de cateter de Swan-Ganz no pósoperatório (Tabela 7).

Comparando-se os pacientes com bacteremia primária, aqueles com infecção sem bacteremia e os que não se infectaram, não houve diferença estatisticamente significante em relação às variáveis relacionadas na Tabela 8.

\section{COMENTÁRIOS}

A incidência de eventos infecciosos nos pacientes cirúrgicos necessitando de suporte circulatório mecânico com balão intra-aórtico mostrou-se elevada $(78,2 \%)$ em comparação com outras séries descritas na literatura $1-3,5,8,9,11,13$, embora estas 
BORGES, L. H. B.; CAMARGO, L. F. A.; STRABELLI, T. M. V.; UIP, D. E.; AULER JÚNIOR, J. O. C. - Contrapulsação em operação cardíaca: análise retrospectiva da incidência de infecção. Rev. Bras. Cir. Cardiovasc., 9 (2): 88-94, 1994

TABELA 7

DIVERSAS VARIÁVEIS COMPARADAS ENTRE OS PACIENTES INFECTADOS E NÃO INFECTADOS. OS DADOS PARA T.S.O.; C.E.C.; DURAÇÃO DO BIA, DO CATETER VENOSO CENTRAL E DE SWAN-GANZ E DE INTERNACCÃO REPRESENTAM O VALOR MÉDIO. T.S.O.: TEMPO DE SALA OPERATORIA; C.E.C.: CIRCULAÇÃO EXTRACORPÓREA

\begin{tabular}{llll}
\hline VARIÁVEL & $\begin{array}{c}\text { INFECTADOS } \\
(N=12)\end{array}$ & $\begin{array}{c}\text { NĀO INFECTADOS } \\
(N=12)\end{array}$ & $P$ \\
\hline T.S.O (minutos) & $485,6 \pm 128,2$ & $406,4 \pm 77,7$ & 0,04 \\
C.E.C (minutos) & $151,9 \pm 63,4$ & $107,8 \pm 43,3$ & 0,09 \\
Operaçōes de Urgência & 44,2 & 25,0 & 0,32 \\
Tempo de permanência & $4,3 \pm 2,01$ & $2,7 \pm 1,5$ & 0,01 \\
Tempo de permanência do Cateter de & $7,5 \pm 10,3$ & $2,5 \pm 1,5$ & $<$ \\
Swan-Ganz Pós-Operatório (dias) & $35,1 \pm 24,1$ & $19,3 \pm 8,0$ & 0,01 \\
Tempo de internação & 20,9 & 8,3 & 0,43 \\
(dias) & & & \\
Reoperaçōes (\%) & & \\
\hline
\end{tabular}

considerassem apenas as infecções diretamente relacionadas ao uso do balão (bacteremias, infecção de ferida).

Esta alta taxa é em parte explicada pelo fato do uso do balão intra-aórtico definir uma população de risco e, portanto, mais propensa à ocorrência de infecções. As características do grupo estudado reforçam esta hipótese: $40 \%$ de operaçōes de urgência, $70 \%$ de ocorrência de complicações no intraoperatório (massagem cardiaca interna ou externa, sangramento importante e dificuldades para saida de circulaçāo extracorpórea), $20 \%$ de reoperações por complicações da primeira operação, duração média das operações acima de 6 horas, tempo de CEC e do uso do BIA prolongados. Estas características são semelhantes às referidas nas outras séries citadas na literatura $1-3,5,8,14$. HSU et alii ${ }^{6}$ demonstraram, estudando 332 pacientes submetidos a transplante cardíaco, maior incidência de infecção nos grupos que usaram BIA $(86 \%)$ ou outros suportes circulatórios mecânicos ("left ventricular assist") em relação aos que usaram coração artificial total $(46 \%)$ ou que não necessitaram destes suportes $(29 \%)$. Por outro lado, o reconhecimento deste grupo como sendo de alto risco pode levar a equipe médica a supervalorizar os diagnósticos infecciosos e iniciar antibioticoterapia mais precocemente.

Sessenta e três por cento dos pacientes apresentaram infecção pulmonar no pós-operatório, taxa muito elevada, quando comparada à taxa geral de pneumonias em pacientes cirúrgicos desta Instituição, no mesmo período $(8 \%$, segundo dados do Grupo de Controle de Infecção Hospitalar). Alguns fatores poderiam explicar estes indices elevados: 1) função ventricular altamente comprometida, sugerida pela própria necessidade do $B \mid A ; 2)$ evolução dos pacientes com intubação orotraqueal prolongada ( 8 dias em média); 3) utilização rotineira de bloqueadores de receptor $\mathrm{H} 2$; 4) superestimação do

TABELA 8

VARIÁVEIS ESTUDADAS NOS PACIENTES COM SUPORTE CIRCULATÓRIO POR BIA DIVIDIDOS EM 3 GRUPOS: AQUELES COM BACTEREMIA PRIMARIA, AQUELES COM INFECCCÃO MAS SEM BACTEREMIA PRIMÁRIA E AQUELES QUE NĀO TIVERAM INFECÇŌEES. T.S.O.; TEMPO DE SALA OPERATÓRIA; C.E.C.; CIRCULAÇĀO EXTRACORPÓREA. OS VALORES REFERIDOS SATO VALORES MEDIOS

\begin{tabular}{|c|c|c|c|c|}
\hline VARIÁVEL & $\begin{array}{c}\text { BACTEREMIA } \\
\text { PRIMARIA } \\
(N=6)\end{array}$ & $\begin{array}{c}\text { S/BACTEREMIA } \\
\text { PRIMÁRIA, } \\
\text { COM INFECÇÃO } \\
(N=37)\end{array}$ & $\begin{array}{c}\text { S/INFECÇĀO } \\
(N=12)\end{array}$ & $P$ \\
\hline Duração do BIA (dias) & 3,3 & 4,5 & 2,7 & $>0,05$ \\
\hline Tempo de cateter após operação (dias) & 11,8 & 20,0 & 4,8 & $>0,05$ \\
\hline Óbitos & 33,3 & 32,4 & 8,3 & $>0,05$ \\
\hline T.S.O. & 426 & 494 & 406 & $>0,05$ \\
\hline (minutos) & 129 & 155 & 107 & $>0,05$ \\
\hline C.E.C. (minutos) & 33,3 & 45,9 & 25,0 & $>0,05$ \\
\hline Operaçāo de Urgência (\%) Reoperaçōes & 16,7 & 21,6 & 8,3 & $>0,05$ \\
\hline
\end{tabular}


diagnóstico de pneumonia frente a infiltrados pulmonares de natureza cardiogênica. O tempo médio de aparecimento das pneumonias foi relativamente precoce, em torno de 3 dias após a operação. Por outro lado, HSU et alii ${ }^{6}$ mostraram, em seus pacientes submetidos a transplante cardiaco, que o índice de pneumonia foi apenas pouco superior no grupo que necessitou suporte circulatório por BIA $(19 \%)$ em relação ao grupo que usou (13\%).

A taxa de infecção do local de inserção do BIA $(7 \%)$ é relativamente alta, se for comparada à taxa geral de infecção da ferida cirúrgica no InCor, em torno de $3 \%$ (dado do Grupo de Controle de Infecção Hospitalar - 1993). Entretanto, está dentro da variação de taxas nos diversos relatos $3,4,8-12$, que foi de $2,6 \%$ a $13,5 \%$. Frente ao número relativamente pequeno de pacientes em nosso estudo, este dado apenas sugere a necessidade de uma vigilância mais rigorosa, avaliando se há uma tendência à manutenção de taxa endêmica alta. A média de uso do BIA foi similar entre os grupos de pacientes com e sem infecção do local de inserção (4 dias contra 3,9 dias). Este dado está concordante com uma série de 733 pacientes ${ }^{8}$ em que se estudaram as complicações relacionadas ao uso do BIA, quando a taxa de infecção no local de inserção não esteve relacionada ao tempo de permanência, embora esta variável aumente significativamente a incidência de febre e bacteremia. Não houve inserçōes transtorácicas do BIA nesta série de pacientes, mas em outros estudos 5,12 esta modalidade esteve relacionada à maior incidência de mediastinite $(2.5 \%$ e $3,0 \%)$.

A taxa de bacteremia primária foi alta (10\%), embora sua interpretação deva ser cuidadosa, já que foram utilizados critérios clínicos para seu diagnóstico, não havendo confirmação etiológica em nenhum dos casos. KANTROWITZ et alii ${ }^{8}$ mostraram $12(1,4 \%)$ casos de bacteremias em 733 pacientes recebendo suporte circulatório com BIA.

A análise dos fatores de risco não mostrou diferença estatisticamente significante entre o grupo de pacientes com bacteremia primária e aquele de pacientes não infectados em relação ao tempo de uso do BIA e também em relação ao tempo de uso de cateter venoso central após a operação, contradição que pode ser devida ao pequeno número de pacientes em ambos os grupos. Além disso, devido à natureza retrospectiva do estudo, nāo foi possivel estabelecer uma relação definitiva entre a presença do BIA e a ocorrência de infecção na corrente sangüínea, devendo ser realizado estudo prospectivo com maior número de pacientes e com padronização da coleta das culturas de sangue e de cultura quantitativa da ponta do BIA.

A média de tempo para diagnóstico de bacteremia primária foi de 2 dias após a inserção do BIA, bastante curta, sendo possivel que este diagnóstico esteja superestimado. Por outro lado, o início de antibioticoterapia para as demais infecções foi precoce (3,3 dias), o que pode ter contribuído para a não confirmação das bacteremias e mesmo para a diminuição do número de casos.

Com relação à análise de fatores de risco para todas as infecções, podemos considerar que o tempo de sala operatória $(p=0,04)$ e o tempo de circulaçāo extracorpórea $(p=0,09)$ são fatores preditivos de gravidade no pós-operatório e, portanto, indicativos de risco aumentado para infecção. Neste estudo, operações com duração em torno de 7,8 horas e com tempo de circulação extracorpórea em torno de 2,5 horas foram altamente associadas à infecção no pós-operatório. Entretanto, sabe-se que estas variáveis não são exclusivas para pacientes em uso de balão intra-aórtico. Mesmo assim, com estes dados e com a alta freqüência de infecções pulmonares no grupo como um todo, pode-se estudar a adoção de medidas prolifáticas para este subgrupo específico.

O tempo de uso do BIA e do cateter de SwanGanz, que apresentaram diferença estatisticamente significante entre infectados e não infectados, são provavelmente indicadores de gravidade, ao invés de fatores associados à ocorrência de infecçōes no pós-operatório, visto que predominaram as infecçōes pulmonares.

Em resumo, mostramos uma incidência relativamente elevada de infecçōes em pacientes submetidos a contrapulsação, principalmente pelo fato de se tratar de população de risco. Como a maioria das infecçōes foi pulmonar, pode-se propor a adoção de medidas profiláticas mais rigorosas, particularmente para os pacientes com tempo de sala operatória maior que 7,8 horas e tempo de circulação extracorpórea maior que 2,5 horas (como, por exemplo, evitar o uso de bloqueadores $\mathrm{H} 2$ e descontaminaçāo seletiva do trato gastrintestinal). Requer-se, também, uma vigilância especifica das infecções da corrente sangüínea com a coleta sistemática de hemoculturas, uma vez que o uso de critérios clínicos ou hemodinâmicos, pelos aspectos multifatoriais envolvidos (drogas vasoativas, ventilação mecânica), é provavelmente insuficiente. 
BORGES, L. H. B.; CAMARGO, L. F. A.; STRABELLI, T. M. V.; UIP. D.; AULER JÚNIOR, J. O. C. - Intra-aortic balloon pumping: incidense of complications related to its insertion. Rev. Bras. Cir. Cardiovasc., 9 (2): 88-94, 1994.

ABSTRACT: The technological advances of recent years have permitted a wide range of uses for intraaort balloon pumping (IABP) to support acute cardiac failure in many clinical condition. In spite of this, some complications related to its insertion, duration of use and location have been described. The aim of his study was to investigate the incidence of infections in severely compromised postoperative cardiac patients undergoing IABP therapy. Between January 1990 and July 1992, the records of 97 patients that required IABP support after cardiac surgery were reviewed, but only 55 met the criteria to be included. The analysis consited of: incidence of infections, culture results, type and duration of by-pass and surgery duration of intravascular catheterization and clinical outcome. The following sites of infection were considered: lung, urine, blood, surgical incision and IABP catheter insertion. The mean time of procedures were: IABP $=3.9 \pm 2.01$ days surgery an by-pass equal to 8.0 and 2.5 hours respectively. We observed a high incidence of infection in these patients, mainly pneumonia $(63.6 \%)$. The IABP insertion infection rate was $7.0 \%$ higher than our general postoperative wound infection rate $(3.0 \%)$. Although this relatively higher incidence of infection may not directly relate to the martality rate, we suggest that these patients must have extremely careful surveillance for ocurrence of infections and possible prophylactic measures regarding pulmonary infections.

DESCRIPTORS: intra-aortic balloon pump, complications, infectious; counterpulsation in heart surgery.

\section{REFERÊNCIAS BIBLIOGRÁFICAS}

ALVAREZ, J. M.; GATES, R.; ROWE, D.; BRADY, P. W. - Complications from intraaortic balloon counterpulsation: a review of 303 cardiac surgical patients. Eur. J. Cardiothorac. Surg., 6: 530-535, 1992.

ETCHANINOFF, H.; DIMAS, A. P.; WHITLOW, P. L. Complications associated with percutaneous placement and use of intraaortic balloon counterpulsation. Am. J. Cardiol., 71: 328-332, 1993.

FREED, P. S.; WASFIE, T.; ZADO, B.; KANTROWITZ, A. - Intraaortic balloon pumping for prolonged circulatory support. Am. J. Cardiol., 61: 554-557. 1988.

4

HARVEY, J. C.; GOLDSTEIN, J. E.; MCCABE, J. C.; HOOVER, E. L.; GAY Jr., W. A.; SUBRAMANIAN, V. A. - Complications of percutaneous intra aortic balloon pumping. Circulation, 64 (Supl. 2): 114-117, 1981.

5 HAZELRIGg, S. R.; AUER, J. E.; SEIFERT, P. E. Experience in 100 transthoracic balloon pumps. Ann. Thorac. Surg., 54: 528-532, 1992.

6 HSU, J.; GRIFFITH, B. P.; DOWLING, R. D.; KORMOS, R. L.; DUMMER, J. S.; ARMITAGE, J. M.; ZENATI, M.; HARDESTY, R. L. - Infections in mortally ill cardiac transplant recipients. J. Thorac. Cardiovasc. Surg., 98: 506-509, 1989.
7 KANTROWITZ, A.; TJONNELAND, S.; FREED, P. S.; PHILLIPS, S. J.; SHERMAN Jr., J. L. - Inicial clinical experience with intraaortic balloon pumping in cardiogenic schock. JAMA, 203: 113-118, 1968.

8 KANTROWITZ, A.; WASFLE, T.; FREED, P. S.; RUBENFIRE, M.; WAJSZEZUK, M. A. - Intraaortic balloon pumping: 1967 through 1982: analysis of complications in 733 patients. A. M. J. Cardiol., 57: 976-983, 1986.

9 LAZAR, J. M.; ZIADY, G. M.; DUMMER, S. J.; THOMPSON, M.; RUFFNER, R. J. - Outcome and complications of prolonged intraaortic balloon counterpulsation in cardiac patients. Am. J. Cardiol., 69: 955-958, 1992.

10 McCABE, J. C.: ABEL, R. M.; SUBRAMANIAN, V. A.; GAY. W. A. - Complications of intraaortic balloon insertion and counterpulsation. Circulation, 57: 769773,1978

11 MCENANY, M. T.; KAY, H. R.; BUCKLEY, M. J.; DAGGETT, W. M.; ERDMANN, A. J.; MUNDTH, E. D.; RAO, R. S.; DeTOEUF, J.; AUSTEN, W. G. Clinical experience with intraaortic balloon pump support in 728 patients. Cardiovasc. Surg., 58 (Supl. 1): $124-132,1977$

12 McGEEHIN, W.; SHEIKH. F.; DONAHOO, J. S.; LECHMAN, M. J.; MAC VAUGH, H. - Transthoracic 
BORGES, L. H. B.; CAMARGO, L. F. A.; STRABELLI, T. M. V.; UIP, D. E.; AULER JÚNIOR, J. O. C. - Contrapulsação em operação cardiaca: análise retrospectiva da incidência de infecção. Rev. Bras. Cir. Cardiovasc., 9 (2): 88-94, 1994

intraaortic balloon pump support: experience in 39 patients. Ann. Thorac. Surg., 44: 26-30, 1987.

13 PENNINGTON, D. G.; SWARTZ, M.; CODD, J. E.; MERJAVY, J. P.; KAISER, G. C. - Intraaortic balloon pumping in cardiac surgical patients: a nine year experience. Ann. Thorac. Surg., 36: 125-121, 1983.

14 PIFARRE, R.; SULLIVAN, H.; MONTOYA, A.; BAKHOS M.; GRIECO, J.; FOY, B. K.; BLAKEMAN, B.; CONSTANZO-NORDIN, M.R.; ALTERGOTT, R.;
LONCHYNA, V.; CALANDRA, D.; HINKAMP, T.; GRADY, K. L.; BARTLETT, L. - Comparison of results after heart transplantation: mechanically supported versus nonsupported patients. J. Heart Lung Transplant, 11: 235-239, 1992.

15 WASFIE, T.; FREED, P. S.; RUBENFIRE, M.; WAJSZCZUK, W.; REIMANN, P.; BROZYNA, W.; SCHORK, M. A.; KOZLOWSKI, J.; KANTROWITZ, A. - Risk associated with intraaortic balloon pumping in patients with and without diabetes mellitus. Am. J. Cardiol., 61: 558-562, 1988. 